\title{
Enediyne-activated, EGFR-targeted human $\beta$-defensin 1 has therapeutic efficacy against non-small cell lung carcinoma
}

\author{
Wen-juan $\mathrm{Liu}^{1} \cdot \mathrm{Kun}-\mathrm{li} \mathrm{Zhu}^{2} \cdot$ Jian $\mathrm{Xu}^{3} \cdot$ Jia-lin Wang ${ }^{1} \cdot \mathrm{Hui} \mathrm{Zhu}^{2}$
}

Received: 2 January 2018 / Revised: 7 June 2018 / Accepted: 28 June 2018 / Published online: 11 September 2018

(c) United States \& Canadian Academy of Pathology 2018

\begin{abstract}
Human $\beta$-defensins contain an oncolytic motif that binds to tumor cell membranes and mediate permeabilization, rapid induction of cytolysis, and apoptosis. Previous studies have indicated that a fragment of the mature human $\beta$-defensin-1 (HBD1) peptide (DF) has antitumor properties. While targeted drug treatments using fusion proteins have been shown to increase drug efficacy, this phenomenon has not been studied for this defensin. Thus, in this study, we designed and prepared a fusion protein containing this HBD1 fragment and an epidermal growth factor receptor (EGFR)-targeting oligopeptide (Ec) as well as lidamycin (LDM), an extremely potent cytotoxic antitumor antibiotic, which consists of an apoprotein (LDP) and a highly active enediyne (AE). The fusion protein (Ec-LDP-DF) and its enediyne-integrated fusion protein (Ec-LDP(AE)DF) were then purified and used to treat lung carcinoma cells in culture as well as lung carcinoma xenograft mouse models. The multifunctional fusion protein Ec-LDP-DF was shown to effectively bind to EGFR-expressing tumor cells. Furthermore, the enediyne-energized Ec-LDP(AE)-DF analog exhibited extremely potent cytotoxicity in NSCLC cell lines and an $\mathrm{IC}_{50}$ less than $10^{-10} \mathrm{~mol} / \mathrm{L}$. Ec-LDP(AE)-DF also significantly inhibited the growth of human carcinoma A549 and H460 xenografts in athymic mice at well-tolerated doses. Treatment resulted in cell cycle arrest and apoptosis in a dose-dependent manner. EGF-stimulated EGFR phosphorylation was also abolished by Ec-LDP(AE)-DF. In summary, our understanding of the role of defensins in cancer development and progression is continually expanding, and Ec-LDP(AE)-DF is a promising cancer cell-targeting agent for NSCLC.
\end{abstract}

These authors contributed equally: Wen-juan Liu, Kun-li Zhu

Electronic supplementary material The online version of this article (https://doi.org/10.1038/s41374-018-0109-5) contains supplementary material, which is available to authorized users.

Hui Zhu

drzhuhui@163.com

1 Shandong Provincial Key Laboratory of Radiation Oncology, Shandong Cancer Hospital and Institute, Shandong Cancer Hospital affiliated to Shandong University, Shandong Academy of Medical Sciences, Jinan 250117, China

2 Department of Radiation Oncology, Shandong Cancer Hospital affiliated to Shandong University, Shandong Academy of Medical Sciences, Jinan 250117 Shandong Province, China

3 Institute of Medicinal Biotechnology, Chinese Academy of Medical Sciences and Peking Union Medical College, Beijing 100050, China

\section{Introduction}

Human defensins are small cationic peptides produced by epithelial cells and neutrophils in two genetically distinct forms, $\alpha$ - and $\beta$-defensins [1-3]. Previous studies have shown that human $\beta$-defensins, in addition to playing a role in the innate immune system, have antibacterial properties, anti-viral activity, and tumor cell cytotoxicity. In fact, compared with traditional chemotherapeutics, it has been shown to be more difficult for cancer cells to develop resistance to defensins [4]. Additionally, they show no immunogenicity and are resistant to proteolysis [5]. Human $\beta$-defensin-1 (HBD1) appears to kill tumor cells via a unique mechanism that involves membrane lysis and DNA manipulation [6, 7]. Gene therapy with HBD1 also inhibits tumor growth in renal carcinoma and prostate cancer [7]. Recently, the plant defensin $\mathrm{NaD} 1$ and human $\beta$-defensin 3 (HBD3) were both shown to induce tumor cell lysis by directly binding to the plasma membrane phosphoinositide, phosphatidylinositol 4,5-bisphosphate $(\mathrm{PI}(4,5) \mathrm{P} 2)$ [8]. These data not only identify an evolutionary conservation of 
Fig. 1 Preparation of Ec-LDP (AE)-DF and determination of its enediyne-integrated analog Ec-LDP(AE)-DF. a The diagram indicates the components of the Ec-LDP-DF fusion protein and its reconstituted enediyneintegrated analog Ec-LDP(AE)DF. b Expression analysis of EcLDP-DF protein by $12 \%$ SDSPAGE. Lane 1, molecular weight marker; Lanes 2-4, expression analysis of Ec-LDP$\mathrm{DF}$. The fusion protein is indicated using arrow. $\mathbf{c}$ Western blot detection of the Ec-LDP-DF fusion protein using mouse antiHis tag monoclonal antibody (1:1000 dilution) and HRPconjugated goat anti-mouse IgG (1:2000 dilution). d Reversephase HPLC determination for the enediyne-integrated analog Ec-LDP(AE)-DF using a Vydac C4 300A column at $340 \mathrm{~nm}$
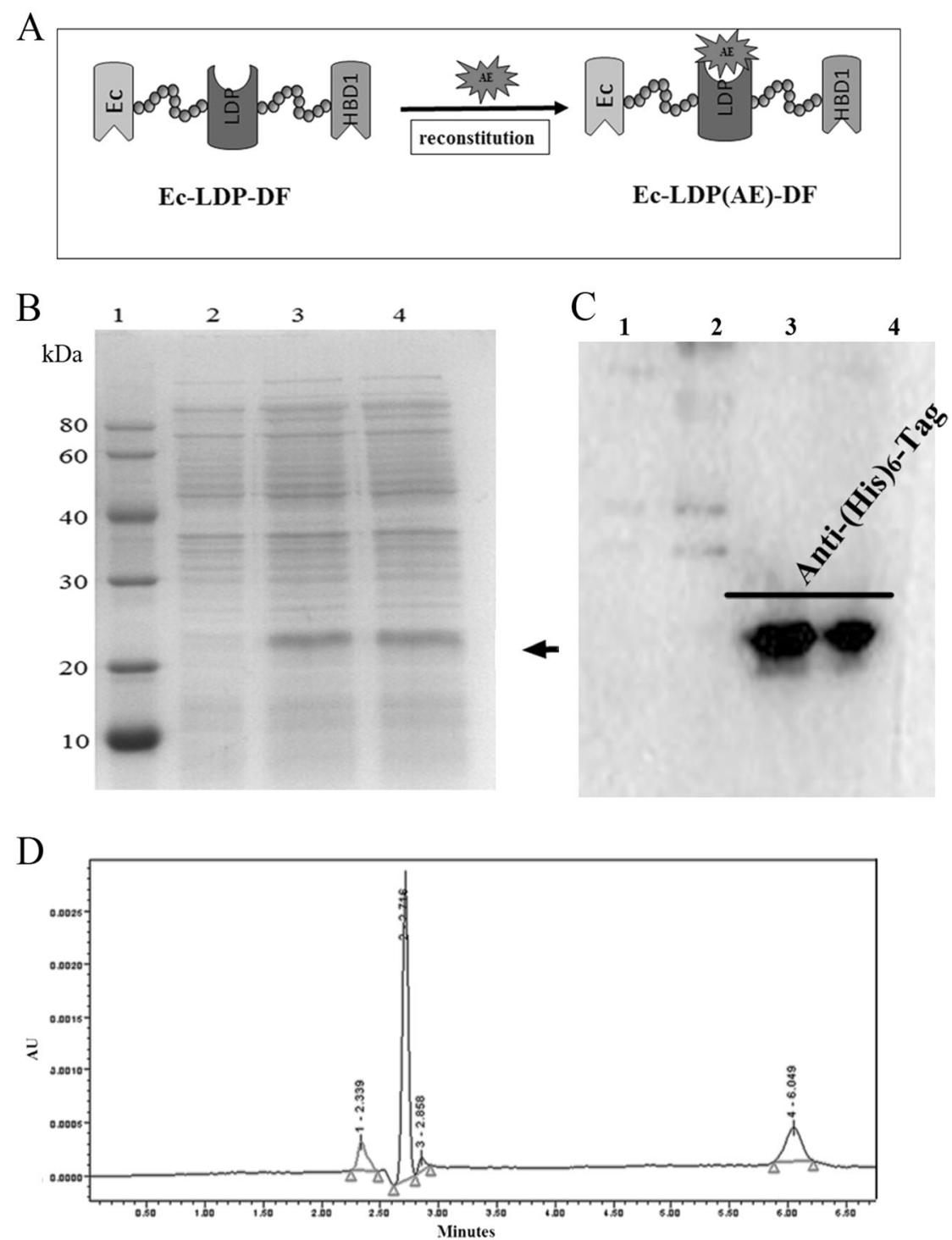

defensin structure and function for lipid binding, but also suggest that cell membrane-bound defensins could be exploited as novel multifunctional therapeutics.

Lidamycin (LDM, also called C-1027) is an antitumor antibiotic with an extremely potent cytotoxicity [9]. The LDM molecule consists of an active enediyne chromophore (AE, $843 \mathrm{Da}$ ), which is responsible for the molecule's strong cytotoxic properties, and a non-covalently bound apoprotein (LDP, 10,500 Da), which acts as a hydrophobic domain to stabilize and protect the enediyne chromophore [10]. As reported, AE and LDP can be dissociated and reassociated in vitro under certain conditions, and the reconstituted LDM has similar properties as that of natural LDM $[11,12]$. Notably, LDM has also been widely used as an effector molecule or "warhead" in various fusion proteins, and its tandem expression with other therapeutics appears to synergistically affect cancer cell function and tumor growth.
Furthermore, to target LDM and/or other treatments, the binding substrates of various cell surface receptors have been employed. Epidermal growth factor receptor (EGFR), for example, is overexpressed in many human tumors and has been used to develop targeted therapeutics in non-small cell lung carcinoma (NSCLC) [11, 13]. Indeed, an oligopeptide (Ec) derived from EGF binds to EGFR in lung cancer cells, making it an ideal guiding molecule in the preparation of EGFR-targeted fusion proteins [14]. While these cancer cell-targeting fusion proteins have been used in multiple studies, to date, these techniques have not been used in the application of defensin as a cancer therapeutic.

In the present study, a pair of EGFR-targeted, HBD1based fusion proteins, Ec-LDP-DF and Ec-LDP(AE)-DF, were constructed. In these fusion proteins, Ec is the EGFR binding peptide and HBD1 is the effector agent. The apoprotein LDP is employed as the scaffold of the fusion 
protein and the "pocket" for the insertion of enediyne AE. Furthermore, the recombinant EGFR-targeted defensin EcLDP-DF and its enediyne-integrated analog Ec-LDP(AE)DF were analyzed and evaluated for their EGFR targeting capability in NSCLC, antitumor efficacy, and related mechanism of action. The purpose of this study was to use known fusion protein expression techniques (EGFR oligonucleotide and LDM) to target HBD1 expression to cancer cells and to evaluate the potential applications of this therapeutic using both in vitro and in vivo methods.

\section{Materials and methods}

\section{Cell culture}

Human lung carcinoma A549, H520, PG-BE1, and H460 cells were cultured as reported previously [15]. Human cancer cell lines A549, H520, and H460 were purchased from the American Type Culture Collection (ATCC), these cell lines were tested by STR analysis by the China Center for Type Culture Collection (CCTCC) in 2013. The human lung cell line PG-BE1 was a kind gift from Professor Hongwei $\mathrm{He}$ (Institute of Medicinal Biotechnology, Chinese Academy of Medical Sciences and Perking Union Medical College).

\section{Construction of pET-Ec-Idp-DF}

The full gene of the recombinant protein Ec-LDP-DF (from 5' to $3^{\prime}$ ) consists of the C-terminal C-loop of EGF (22 amino acids), apoprotein LDP (110 amino acids), and HBD1(DF) (36 amino acids) (Fig. 1a). A G4S linker was inserted into the space between the EGF C-loop and LDP coding sequences as well as between those of LDP and DF. After PCR and DNA cloning, the resulting 546-bp fragment was digested by $\mathrm{NdeI} / \mathrm{XhoI}$ and inserted into the pET30a expression vector to generate the pET-Ec-ldp-DF plasmid [16].

\section{Expression and purification of the fusion protein and its enediyne-integrated active analog}

The pET-Ec-ldp-DF plasmid was transformed into Escherichia coli strain BL21 (DE3) star (Novagen). After overnight culture, bacteria were grown at $37^{\circ} \mathrm{C}$ until the $\mathrm{OD}_{600}$ reached 1.0. Gene expression was induced with the addition of $1 \mathrm{mmol} / \mathrm{L}$ IPTG, followed by incubation at $37{ }^{\circ} \mathrm{C}$ for $8 \mathrm{~h}$. The proteins were purified by affinity chromatography via the plasmid's C-terminal $6 \times$ His-tag (HisTrap HP, GE Healthcare) according to the manufacturer's instruction. The purified proteins were refolded using stepwise dialysis as reported [12], and the purity of the Ec-LDP-hBD1 protein was analyzed by high-performance liquid chromatography (HPLC) on a S3000 column. The Ec-LDP and LDP proteins were produced as reported in our previous study [11].

In addition, the enediyne-integrated Ec-LDP(AE)-DF fusion protein was prepared and purified. The AE of LDM was separated using a C4 column (GE Healthcare) with a $22 \%$ acetonitrile in $0.05 \%$ trifluoroacetic acid mobile phase. The AE-containing solution was added to Ec-LDP-DF/PBS $(10 \mathrm{mM}, \mathrm{pH} 7.4)$ and incubated at $4{ }^{\circ} \mathrm{C}$ overnight while shaking. Then, the free AE was removed with a Sephadex G-75 column (GE Healthcare). The enediyne-integrated EcLDP(AE)-DF fusion protein was confirmed by reversephase HPLC using a Vydac C4 300A column (Grace). Absorbance at $340 \mathrm{~nm}$ was also determined.

\section{Cancer cell binding specificity}

The binding specificity of the Ec-LDP-DF fusion protein was analyzed in A549 and H460 cells using fluorescence microscopy as previously reported $[17,18]$. To quantitatively compare the target cell binding affinity of each protein sample, we used a fluorescence-activated cell sorting (FACS)-based saturation binding assay. The data were analyzed with Prism 5.0 software (GraphPad Software).

\section{Cell viability assay}

Cells were plated in 96-well plates at $2 \times 10^{3}$ cells per well. The cells were serum-starved for $8 \mathrm{~h}$ and further incubated with various concentrations of Ec-LDP-DF, Ec-LDP, LDP, or HBD1 for $24 \mathrm{~h}$ at $37^{\circ} \mathrm{C}$. After adding $10 \mu \mathrm{L}$ CCK-8 solution (Dojindo, Kumamoto, Japan) to $100 \mu \mathrm{L}$ of culture media according to the manufacturer's instructions, the optical density was measured at $450 \mathrm{~nm}$.

To quantify cell growth, MTT assays were performed as described [13, 19]. Briefly, each cancer cell line was plated in 96-well plates (4000 cells/well). After incubating overnight, cells were treated with LDM, Ec-LDP(AE)-DF, or cisplatin at varying concentrations $\left(1 \times 10^{-7}, 1 \times 10^{-8}, 1 \times\right.$ $10^{-9}, 1 \times 10^{-10}, 1 \times 10^{-11}, 1 \times 10^{-12}, 1 \times 10^{-13} \mathrm{M}$, respectively) and continuously incubated for $48 \mathrm{~h}$. The MTT reagents $(20 \mu \mathrm{L}, 5 \mathrm{mg} / \mathrm{mL})$ were then added to each well and incubated for an additional $4 \mathrm{~h}$. After removal of the supernatant, $150 \mu \mathrm{L}$ DMSO was added to each well. The optical density (OD) at $570 \mathrm{~nm}$ was determined with a microplate reader (Thermo Fisher Scientific, USA). Untreated cells served as the controls. The relative cell viability (\%) compared with the control was calculated using the formula: cell viability $(\%)=[($ Asample - Ablank)/(Acontrol-Ablank) $] \times 100$. The IC50 values represent the drug concentration resulting in $50 \%$ growth inhibition. Results were derived from three independent experiments. 


\section{Flow cytometric analysis}

A549, H520, and H460 cells were treated with $0.1,0.5$, or $1 \mathrm{nmol} / \mathrm{L}$ of Ec-LDP(AE)-DF for $48 \mathrm{~h}$. Then, the cells were harvested by trypsinization, washed with PBS, and fixed with cold $70 \%$ ethanol at $-20{ }^{\circ} \mathrm{C}$ for $24 \mathrm{~h}$. Cells were centrifuged at $1000 \mathrm{rpm}$ for $5 \mathrm{~min}$ and the cell pellet was washed three times with PBS and resuspended in $0.5 \mathrm{~mL}$ PBS with $50 \mu \mathrm{g} / \mathrm{mL}$ propidium iodide (PI) and $100 \mu \mathrm{g} / \mathrm{mL}$ RNase A. After incubation at $37{ }^{\circ} \mathrm{C}$ for $30 \mathrm{~min}$, the cells were analyzed for fluorescence with a flow cytometer (BD Company) and the percentage of cells in each specific cell cycle phase (G1, S, and G2/M) was determined.

A549, H520, and H460 cells were seeded in 6-well plates at a density of $1 \times 10^{5}$ and incubated for $24 \mathrm{~h}$. After treatment with $0.1,0.5$, or $1 \mathrm{nmol} / \mathrm{L}$ Ec-LDP(AE)-DF for $24 \mathrm{~h}$, the cells were harvested (including the non-adherent cells), washed twice with PBS, and stained with Annexin V-FITC and PI according to the manufacturer's protocol (BD, USA). The fluorescence intensity was measured with a flow cytometer (BD Company).

\section{Animal experiments}

Antitumor experiments were carried out using A549 and H460 xenograft models. Female athymic mice (BALB/c, $\mathrm{nu} / \mathrm{nu}$ ) were purchased from the Institute for Experimental Animals, Chinese Academy of Medical Sciences \& Peking Union Medical College. The study protocols were performed in accordance with the regulations of Good Laboratory Practice for Non-Clinical Laboratory Studies of Drugs issued by the National Scientific and Technologic Committee of People's Republic of China. The treatment and use of animals during the study was approved by the Animal Ethics Committee of the Institute of Medicinal Biotechnology, Chinese Academy of Medical Sciences \& Peking Union Medical College. Epidermoid carcinoma A549 cells and lung cancer H460 cells were respectively suspended in sterile saline $\left(1 \times 10^{7}\right.$ cells $/ \mathrm{mL}$ ) and $200 \mu \mathrm{L}$ of the suspension were inoculated s. c. in the right armpit of each athymic mouse. After 2 weeks, tumors in the donor animals were aseptically dissected and mechanically minced. Blocks of the tumor $\left(2 \mathrm{~mm}^{3}\right.$ in size) were transplanted subcutaneously into athymic mice using a trocar needle. When the tumor size reached approximately $100 \mathrm{~mm}^{3}$ in size, the mice were divided into groups $(n=6)$ and treated with different doses of the sample proteins in $200 \mu \mathrm{L}$ volumes of sterile saline by intravenously injected into the tail vein. A total of two injections were administered with a weekly interval. One group of mice was administered i.v. with physiological saline as a control. Tumor volumes were measured as described previously [18].

\section{Histopathological examination}

The potential toxicity resulting from treatment was monitored by histopathological examination. At the end of the 30 th or 27 th observation day, the athymic mice were euthanized and specimens were taken from the tumor, heart, lung, liver, spleen, and kidney of the group treated with $0.24 \mathrm{mg} / \mathrm{kg}$ Ec-LDP(AE)-DF and the control group. After being fixed in $10 \%$ formalin and embedded in paraffin, histological sections ( $5 \mu \mathrm{m}$ in thickness) were prepared and stained with hematoxylin and eosin (H\&E). The histopathological changes were examined with a Leica microscope.

\section{Western blot analysis}

For protein extraction from tumor tissues, $100 \mathrm{mg}$ of the tumor was put into $1 \mathrm{~mL}$ RIPA lysis buffer (Invitrogen, USA) supplemented with $1 \mathrm{mmol} / \mathrm{L}$ PMSF, followed by homogenization on ice and lysis on ice for $30 \mathrm{~min}$. Extracts were clarified by centrifugation at $10,000 \mathrm{~g}$ for $15 \mathrm{~min}$ at $4{ }^{\circ} \mathrm{C}$, and the total protein was quantitated using a bicinchoninic acid kit (Pierce Biochemicals). A total of $20 \mu \mathrm{g}$ of each protein was separated via $10 \%$ SDS-PAGE and then electroblotted onto polyvinylidene difluoride (PVDF) membranes (Millipore). The membranes were incubated with $1 \%$ BSA for $2 \mathrm{~h}$ at room temperature before incubation overnight at $4{ }^{\circ} \mathrm{C}$ with primary antibodies (diluted 1:1000, Cell Signaling Technology). Then, the membranes were incubated with secondary HRPconjugated antibodies (1:3000 dilution; Cell Signaling Technology) for $1 \mathrm{~h}$ after washing three times with TBST buffer. The specific protein bands were visualized with the Immobilon Western Chemiluminescent HRP Substrate kit (Millipore) and captured with a AI600 imaging system (GE Corp.).

\section{Immunohistochemical analysis}

Immunohistochemical analyses were performed on tumor tissue sections using an antibody specific for $\mathrm{Ki}-67$ as described previously [20]. Stained sections were observed using a microscope, and the images were analyzed using the Leica software system.

\section{Statistical analysis}

Statistical analyses were performed using SPSS software, version 17.0 (SPSS Inc.), or with GraphPad Prism 5.0 (GraphPad Software, Inc.). An unpaired Student's $t$ test or ANOVA test was used to perform statistical comparisons between two groups. The level of significance was set at $p<0.05$. 
Fig. 2 Binding affinity of EcLDP-DF in vitro. a Expression of EGFR in different carcinoma cell lines analyzed by western blotting. b Binding affinity of Ec-LDP-DF to EGFR in A549 and H460 cells. Following FACS, the mean fluorescence intensity was plotted versus protein concentration. c Immunofluorescent binding affinity assay of Ec-LDP-DF in A549 and H460 cells. Red fluorescence (located around the cells) indicates staining with the anti-His-tag antibody (TRITCconjugated second), while blue fluorescence indicates nuclear staining with 4',6-diamidino-2phenylindole (DAPI)
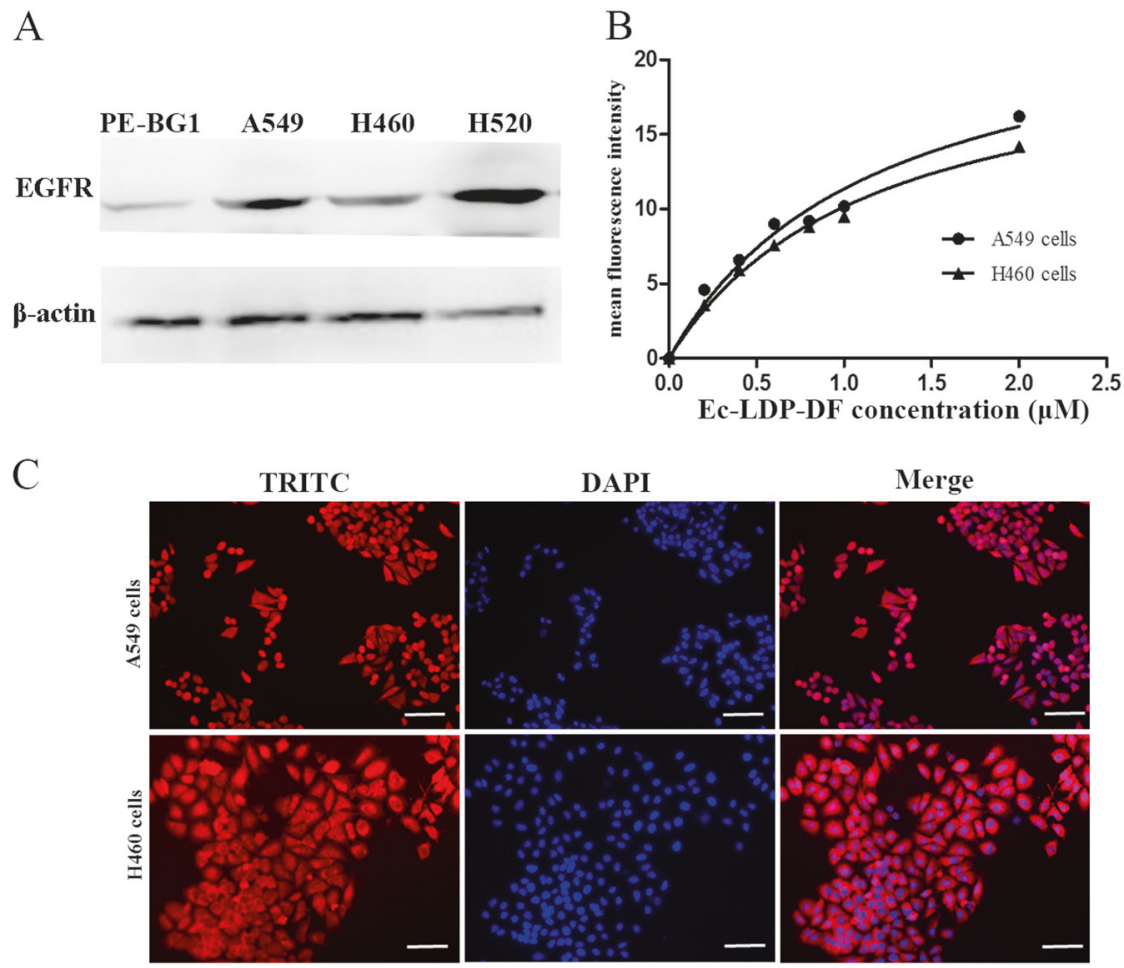

\section{Results}

\section{Construction, preparation, and characterization of EGFR-targeted $\beta$-defensin and its enediyne-integrated analog}

The enediyne-integrated, EGFR-targeted defensin Ec-LDP (AE)-DF was prepared by assembling the active $\mathrm{AE}$ molecule of LDM with Ec-LDP-DF (Fig. 1a). The purity and identity of the Ec-LDP-DF fusion protein were analyzed by $12 \%$ SDS-PAGE (Fig. 1b) and western blot (Fig. 1c). Moreover, the Ec-LDP-DF protein yield from $1 \mathrm{~L}$ of fermentation broth was about $30 \mathrm{mg}$, while that of EcLDP was only about $4 \mathrm{mg}$. The highly active fusion protein Ec-LDP(AE)-DF was prepared by molecular reconstitution of LDP-containing fusion protein Ec-LDP-DF and enediyne $\mathrm{AE}$ derived from natural LDM in vitro. Reverse-phase HPLC shows that the AE molecule was assembled successfully (Fig. 1d).

\section{Binding affinity of the Ec-LDP-DF fusion protein}

Relative EGFR expression was evaluated in PE-BG1, A549, H460, and H520 cells by western blot analysis (Fig. 2a). Anti- $\beta$-actin antibody was used as the quantitative standard. Following treatment, the EGFR targeting ability of the Ec-LDP-DF fusion protein was evaluated using FACS analysis. These data indicate that Ec-LDP-DF was strongly bound to the EGFR-overexpressing A549 cells. In contrast, this binding was weaker for the $\mathrm{H} 460$ cells, which only express low levels of EGFR (Fig. 2b). Ec-LDP-DF protein was also shown to bind specifically to the membrane of EGFR-expressing lung cancer cells (Fig. 2c).

\section{Cytotoxicity of Ec-LDP(AE)-DF in lung cancer cells}

The cytotoxicity of Ec-LDP-DF and Ec-LDP(AE)-DF was tested in four lung carcinoma cell lines, including A549, H460, H520, and PG-BE1 (Fig. 3). In the MTT assay (Fig. 3a), enediyne-integrated Ec-LDP(AE)-DF fusion proteins showed highly potent cytotoxicity in the tested lung cancer cell lines. For A549, H520, H460, and PG-BE1 cells, the IC50 values for Ec-LDP(AE)-DF were $7.3 \times 10^{-12}$, $1.75 \times 10^{-12}, 6.71 \times 10^{-10}$, and $1.09 \times 10^{-10} \mathrm{M}$, respectively (Fig. 3b). Furthermore, Ec-LDP-hBD1 was more potent than cisplatin (DDP) at equivalent concentrations $(p<0.05)$. In the CCK-8 assay (Fig. 3c), Ec-LDP-DF markedly inhibited cancer cell proliferation. The Ec-LDP fusion protein and free LDP and HBD1 were also tested for comparison. At equivalent concentration, Ec-LDP-DF was more potent than Ec-LDP as well as free LDP and HBD1, suggesting that the potency of HBD1 is increased in cancer cells by tethering with Ec-LDP.

Human defensins were previously reported to induce tumor cell lysis by directly binding to the plasma membrane phosphoinositide, PI(4,5)P2 [8]. In the present study, after exposure to $5 \mu \mathrm{M}$ of Ec-LDP-DF for $24 \mathrm{~h}$, the morphology of A549 and H520 cells was significantly changed 
Fig. 3 Cytotoxicity of Ec-LDPDF and Ec-LDP(AE)-DF. a MTT assay of lidamycin and enediyne-energized Ec-LDP (AE)-DF and cisplatin in NSCLC cells. b IC50 values of lidamycin, Ec-LDP(AE)-DF, and cisplatin in NSCLC cells. One-way ANOVA and Dunnett's multiple comparison test were used. $* p<0.05$, $* * * p<0.001$ (Ec-LDP(AE)-DF vs lidamycin or cisplatin). c A549 cell viability after treatment with the Ec-LDP-DF fusion protein (without active enediyne) and Ec-LDP as well as free LDP and HBD1. Experiments were performed in triplicate, and the graph shows the mean and SD
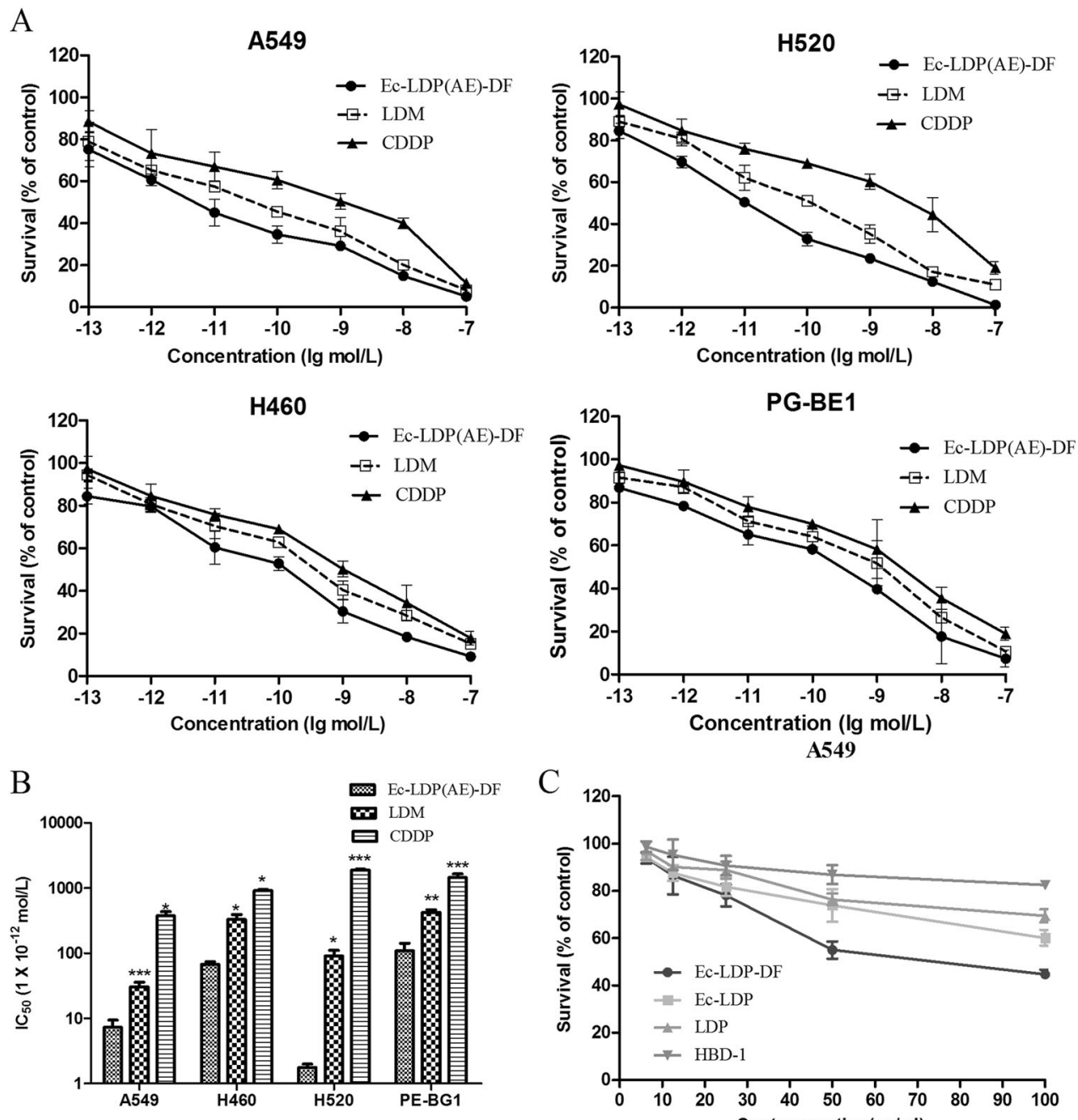

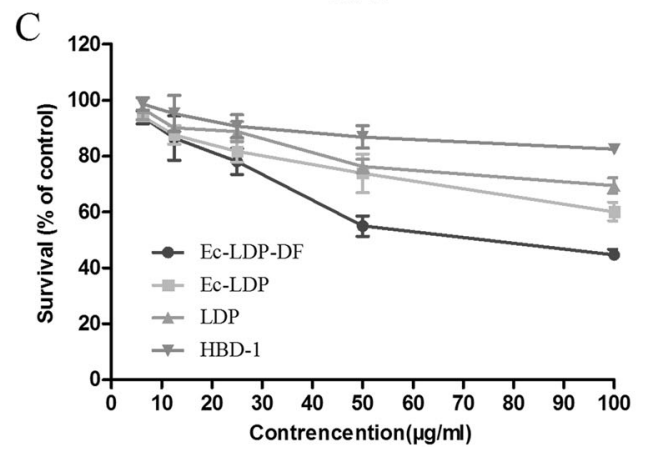

(Figure S1). These cytopathological changes indicate that $\beta$ defensin may exert its cytotoxic effects while being integrated in the form of the fusion protein.

\section{Effects of Ec-LDP(AE)-DF on cell cycle distribution and cell apoptosis}

After treatment with Ec-LDP(AE)-DF for $48 \mathrm{~h}$, cell cycle distribution of NSCLC cells was significantly altered, with many of the cells being arrested in the G2/M phase (Fig. 4a). In this analysis, the percentage of control H520 cells distributed in the $\mathrm{G} 2 / \mathrm{M}$ phase was $12.27 \pm 0.9 \%$, whereas the distribution of cells treated with $0.1,0.5$, and $1 \mathrm{nmol} / \mathrm{L}$ of Ec-LDP(AE)-DF in the G2/M phase was $35.17 \pm 3.2 \%, 42.16 \pm 1.4 \%$, and $65.46 \pm 5.18 \%$, respectively. Similar results were also observed in A549 cells and H460 cells after exposure to Ec-LDP(AE)-DF.

Both the early and late apoptosis ratios increased in H460, A549, and H520 cells treated with Ec-LDP(AE)-DF for $24 \mathrm{~h}$ at different concentrations, as highlighted in the
Annexin V-FITC/PI Apoptosis FACS assays (Fig. 4b). Notably, H460 cells were highly sensitive to Ec-LDP(AE)DF. A prominent increase in early apoptosis was found in A549 cells when treated with $1 \mathrm{nM}$ of the fusion protein, with the ratio reaching $22.67 \pm 2.05 \%$, indicating that EcLDP(AE)-DF is an effective apoptosis-inducing agent in A549 cells. The percentage of apoptotic cells also increased in the H520 cells and H460 cells after treatment with EcLDP(AE)-DF. To characterize the molecular mechanisms involved in the cytotoxicity and apoptosis-induction of EcLDP(AE)-DF, we examined its effects on the expression of apoptosis-related molecules and molecules in the EGFR/pEGFR signaling pathways. As shown in Fig. S2, Ec-LDP (AE)-DF promoted Bax and Bcl-2 activities as well as PARP cleavage, indicating that Ec-LDP(AE)-DF-induced apoptosis may be associated with mitochondrial pathways. Moreover, treatment with Ec-LDP(AE)-DF leaded to significant decreased phosphorylation of EGFR, whereas the expression of inactivated EGFR were not changed. 
A
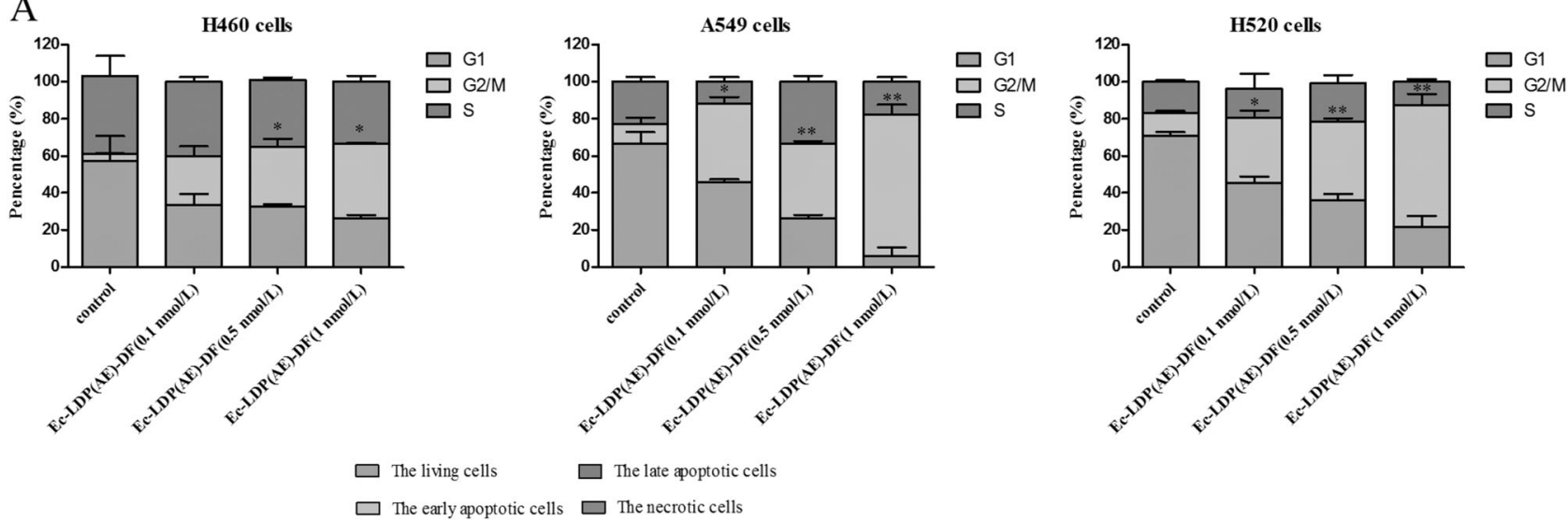

B
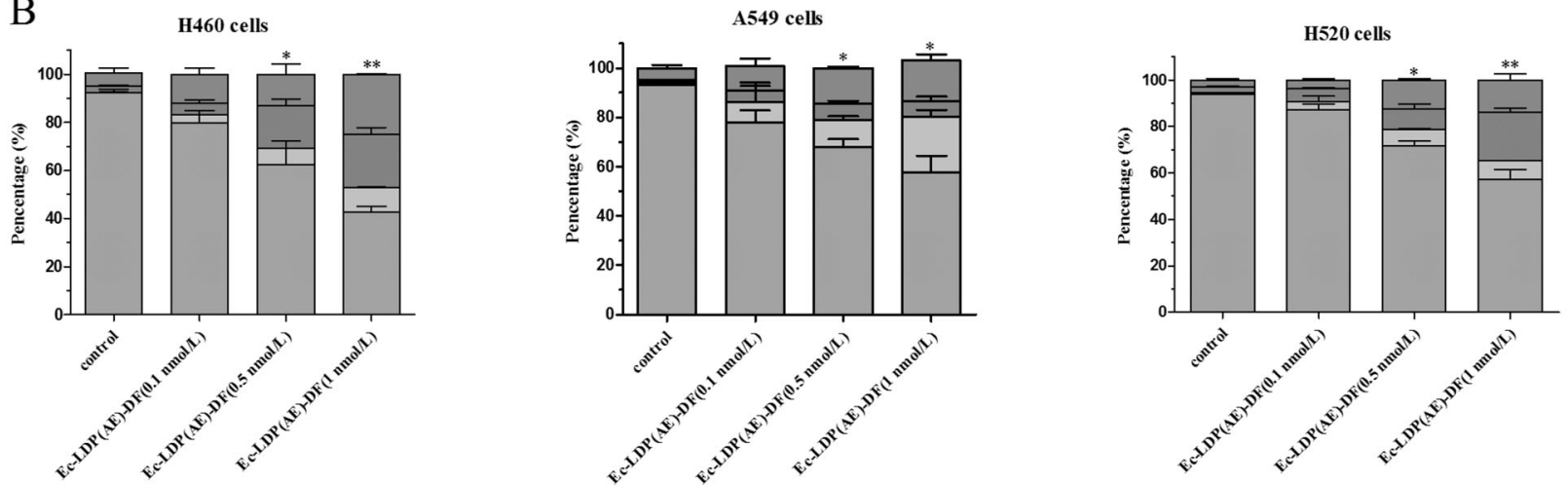

Fig. 4 Effects of Ec-LDP(AE)-DF on cell cycle distribution and cell apoptosis. a NSCLC cells were exposed to Ec-LDP(AE)-DF for $48 \mathrm{~h}$ at the indicated concentrations and cell cycle distribution was determined by flow cytometry after PI staining. b NSCLC cells were treated with

Ec-LDP(AE)-DF for $48 \mathrm{~h}$ at the indicated concentrations and the apoptotic cells were stained by Annexin V-FITC and PI. The apoptosis ratios are the sum of early apoptotic cells and late apoptotic cells. $* * p<0.01$, $* * * p<0.001$ (apoptotic cells of Ec-LDP(AE)-DF vs control)

\section{In vivo therapeutic efficacy of Ec-LDP(AE)-DF}

Therapeutic efficacy of Ec-LDP(AE)-DF was evaluated on human lung carcinoma A549 and H460 xenografts in athymic mice. Following tumor formation in the athymic mice, one group of mice was injected with physiological saline, serving as the vehicle control, while the other groups were treated with LDM $(0.05 \mathrm{mg} / \mathrm{kg})$ or Ec-LDP(AE)-DF $(0.12$ or $0.24 \mathrm{mg} / \mathrm{kg})$, respectively. The applied dosage levels were tolerated by the animals. The mice received a total of two intravenous injections with a 10-day and 18-day interval. As shown in Fig. 5a, the enediyne-integrated EcLDP(AE)-DF fusion protein markedly inhibited tumor growth in the A549 xenograft athymic mice, with the 0.12 and $0.24 \mathrm{mg} / \mathrm{kg}$ treatments resulting in $70 \%$ and $77.4 \%$ suppression of tumor growth, respectively. In comparison, free LDM only inhibited tumor growth by $63 \%$. Ec-LDP (AE)-DF also suppressed the growth of $\mathrm{H} 460$ xenografts by $65.8 \%$ and $76.2 \%$ at doses of 0.12 and $0.24 \mathrm{mg} / \mathrm{kg}$, respectively, while LDM only suppressed growth by $47 \%$. There were no deaths or obvious body weight changes found in the treatment or control groups in either the A549
(Fig. 5c) or H460 (Fig. 5d) xenograft mice. These results indicate that Ec-LDP(AE)-DF is highly effective in treating these types of lung carcinoma xenografts.

Tumor weight was also observed to differ between the control group and the Ec-LDP(AE)-DF-treated groups (Fig. 6a). Immunohistochemical staining indicates that EcLDP(AE)-DF treatment exerted a significant antiproliferative effect on the tumor cells in vivo (Fig. 6b). Notably, no histopathological changes were found in the heart, liver, spleen, lung, or kidney of mice treated with $0.24 \mathrm{mg} / \mathrm{kg}$ Ec-LDP(AE)-DF (Fig. 6c), which indicates that this dosage of the fusion protein was tolerated. To further investigate the antitumor mechanisms of this fusion protein in vivo, we analyzed the phosphorylation and total expression of EGFR in the xenograft tumor tissues by western blot. These results demonstrate that EGFR phosphorylation was significantly reduced in the Ec-LDP(AE)-DF (both $0.24 \mathrm{mg} / \mathrm{kg}$ ) treated tumors when compared with the control groups. Moreover, total EGFR expression was also markedly reduced in the Ec-LDP(AE)-DF (both $0.24 \mathrm{mg} / \mathrm{kg}$ ) treated group (Fig. 6d). 
Fig. 5 In vivo efficacy of EcLDP(AE)-DF. Athymic mice bearing human lung carcinoma A549 (a, c) and H460 (b, d) xenografts were treated, respectively with LDM and EcLDP(AE)-DF intravenously at the specified doses $(n=6)$. Mean tumor volumes for the A549 (a) and H460

(b) xenografts as well as mean body weights (c, $\mathbf{d}$, respectively) of mice in each group are shown. Arrowheads indicate the day of injection (days 8 and 15 for the A549 xenografts; days 7 and 16 for the $\mathrm{H} 460$ xenografts). $* p<0.05, * * p<0.01$
A

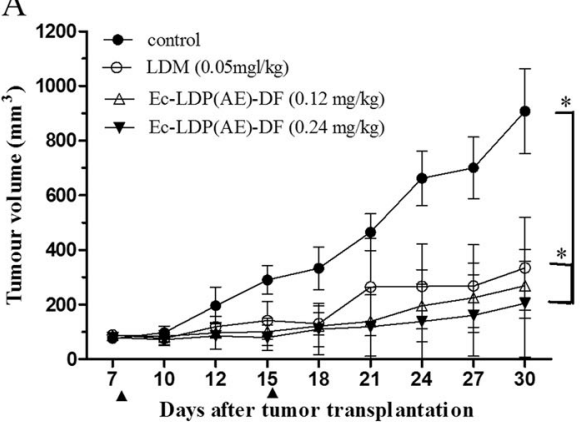

C

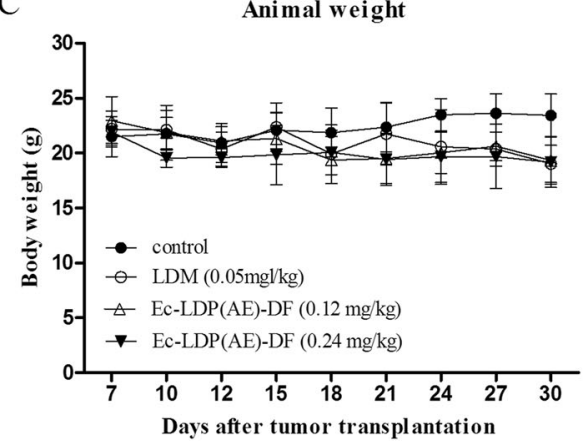

B

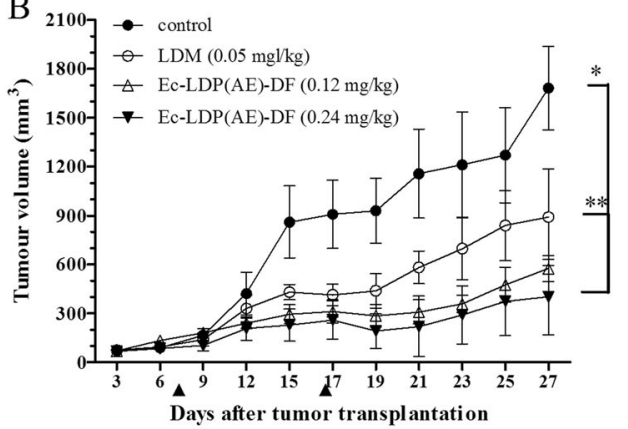

D

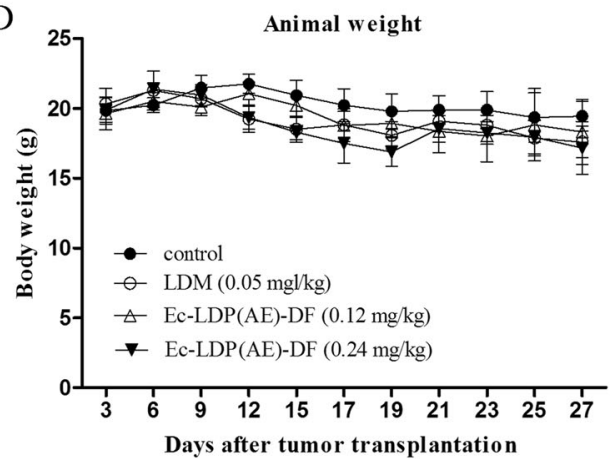

\section{Discussion}

In the current literature, there is growing evidence that defensins can be used as potential agents in cancer treatment [21-23]. Defensins are a major family of antimicrobial peptides that play important roles in innate immunity [24]. Interestingly, defensins are more prone to interact with tumor cells than normal cells, resulting in disruption of their membranes. This preference is likely due to the cationic properties of these proteins, which enable them to interact with the high percentage of anionic molecules, including phosphatidylserine and sialic acid residues, present in the cancer cell membrane $[22,25,26]$. Recently, defensin $\mathrm{NaD} 1$ was shown to induce tumor cell lysis by directly binding to $\mathrm{PI}(4,5) \mathrm{P} 2$ in the plasma membrane [27]. A similar mechanism was also observed for HBD3 [8]. Furthermore, HBD1 has also been should to have antitumor activity. While recent work in the field has greatly enhanced our understanding of defensins, the mechanism underlying the observed HBD1-mediated changes in cancer cell function and tumor progression are largely unknown.

In our laboratory, we have designed a variety of ligandbased, receptor-targeted drugs, including a EGFR-targeted, tuftsin-based fusion protein Ec-LDM-TF [15] as well as an a guided carrier-protein, LDP(AE)-TIMP2 [17, 28] and Ec-LDP-TRAIL [19], which expresses an EGFR oligopeptide and tumor necrosis factor related apoptosisinducing ligand (TRAIL). This has allowed us to study the synergistic therapeutic effects of these fusion proteins. In the present study, a bioactive HBD1-integrated, EGFR- targeting fusion protein (Ec-LDP-DF) was constructed and appears to induce A549 and H460 tumor cells lysis (Fig. S1). Furthermore, this fusion protein also appears to be cytotoxic to other NSCLC cell lines in addition to suppressing tumor growth in two xenograft models.

Targeted drug therapy encompasses a wide variety of strategies. One such strategy employs oligopeptides that recognize particular cell-surface receptors and has been shown to increase drug specificity and lowering systemic toxicity for a variety of drugs [29-31]. Here, we used EGFR-specific oligopeptides (Ec) as the binding molecule in HBD1 fusion proteins on the basis of its specific and potent interaction with EGFR [11, 13, 18, 32]. The resulting genetically engineered fusion protein strongly binds to EGFR present on the tumor cell surface.

Studies have shown that the actual concentration of chemotherapy drugs in tumor cells is minimal, with only $1-2 \%$ of the administered dose reaching the tumor [33-35]. Thus, it is imperative to have a potent cytotoxic payload that is effective at picomolar or nanomolar concentrations. LDM, for example, is extremely cytotoxic to multiple cancer cell types and also has an IC50 value 10-fold lower than that of cisplatin. In addition to its potent cytotoxicity, another attractive property of LDM is its ability to act as a "warhead", whereby the LDP and AE can be dissociated and reconstituted in vitro. This makes the construction of fusion proteins very convenient. It appears that the AEactivated fusion protein created in this study (Ec-LDP(AE)$\mathrm{DF}$ ) is extremely cytotoxic to multiple cancer cell lines and has IC50 values 100-fold lower than that of cisplatin. 
A
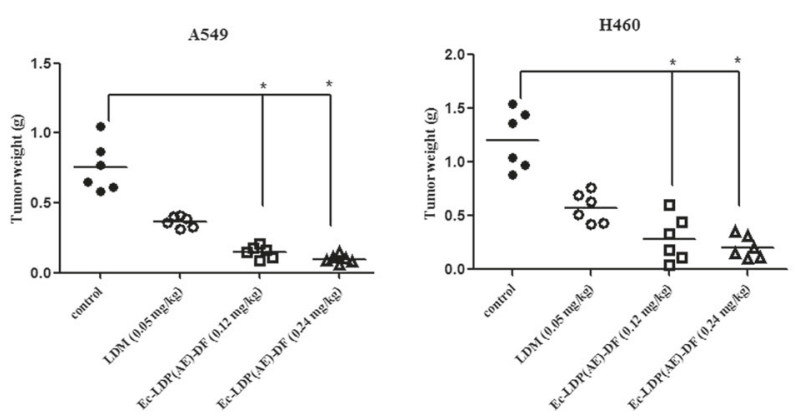

B

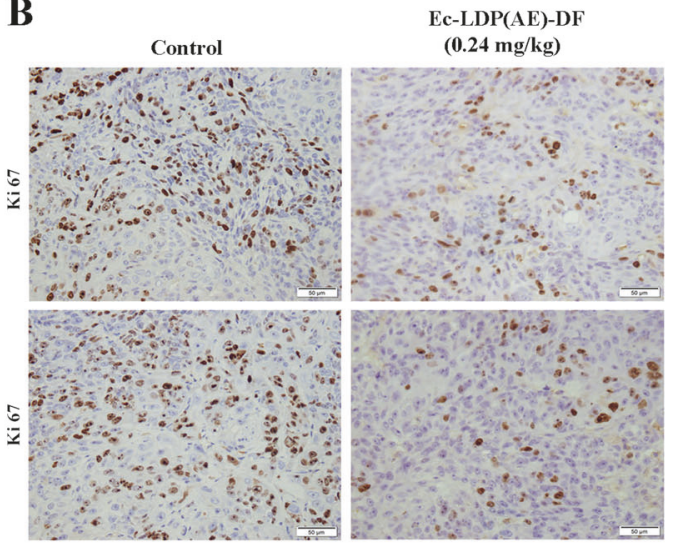

C lung

ung

heart

heart spleen
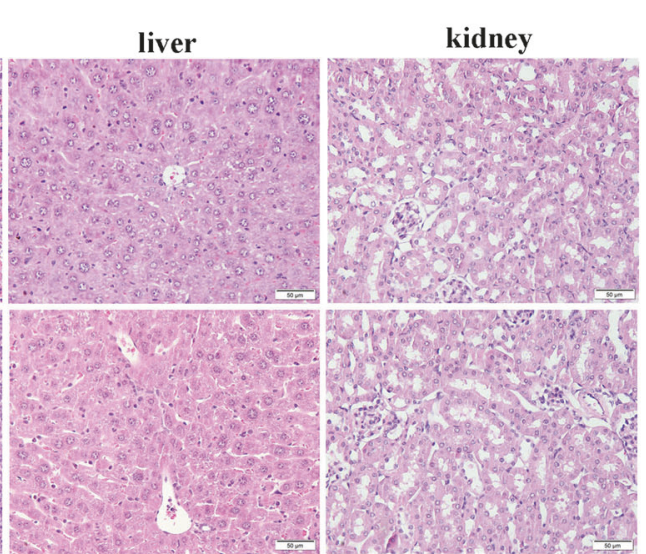

D

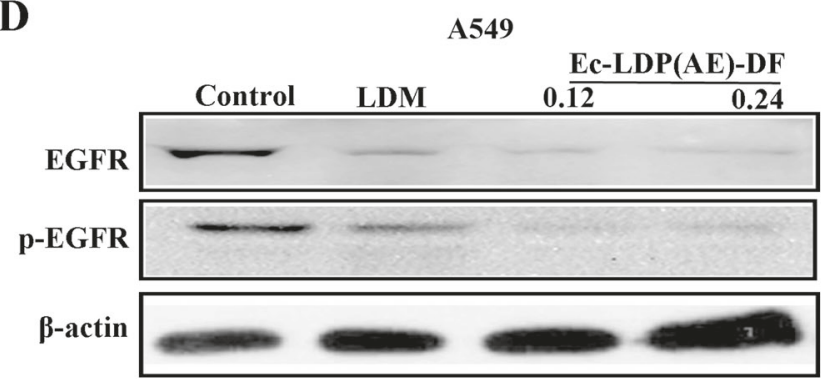

Fig. 6 The molecular effects of Ec-LDP(DF)-AE in lung carcinoma in athymic mice. Athymic mice $(n=6)$ bearing human NSCLC A549 and $\mathrm{H} 460$ xenografts were treated with lidamycin and Ec-LDP(AE)$\mathrm{DF}$ via tail vein injection. a The mean tumor weight after treatment is shown. $\mathbf{b}$ The effect of Ec-LDP(AE)-DF on tumor proliferation marker Ki-67 expression in paraffin-embedded A549 (top) and H460 (down) xenografted tumors was examined by immunohistochemistry (magnification $\times 400$ ). c Histopathological examination of various organs (H\&E staining, magnification $\times 200$ ) of A549 xenograft-bearing mice

Notably, tumor cells overexpressing EGFR, such as A549 and H520, were more sensitive to Ec-LDP(AE)-DF than cells expressing low levels of EGFR, such as H460 and PGBE1, implying that the enhanced potency of Ec-LDP(DF)DF can be attributed to the EGFR-targeted effect in the lung cells overexpressing EGFR. A549 cell proliferation was also markedly inhibited by Ec-LDP-DF, while little effect was found with the Ec-LDP or free HBD1 treatment. These

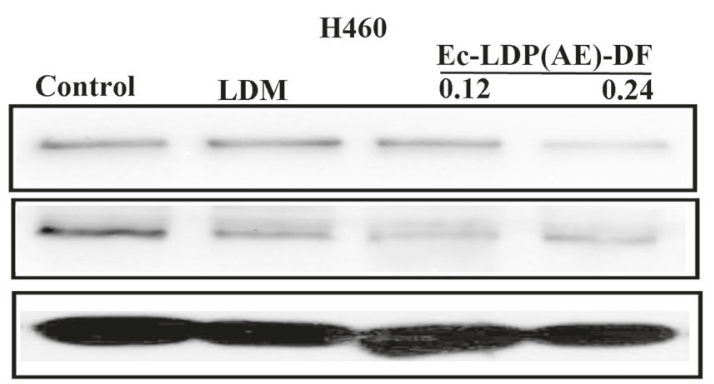

treated with $0.24 \mathrm{mg} / \mathrm{kg}$ of Ec-LDP(AE)-DF. No toxicopathological changes were found in the lung, heart, spleen, liver, or kidney. d The effects of lidamycin and Ec-LDP(AE)-DF on EGFR expression in xenograft tumors. Mice bearing A549 xenografts were sacrificed on day 30. The tumors were isolated and immediately frozen in liquid nitrogen. Total protein was extracted from the tissue, and EGFR phosphorylation and total expression was evaluated by western blot analysis

data indicate that the construction of the fusion protein leads to synergistic effects, whereby the various parts of the protein act together to produce a stronger response. Furthermore, when comparing IC50 values, Ec-LDP(AE)-DF is over 1000 times more cytotoxic than Ec-LDP-DF. This is likely due to the activation of the LDM fragments as studies show that LDM, when activated, inhibits DNA synthesis and causes cellular DNA breakage in cancer cells [9]. 
Indeed, significant levels of cell cycle arrest and apoptosis were observed after the NSCLC cells were exposed to EcLDP(AE)-DF.

Moreover, the enediyne-integrated Ec-LDP(AE)-DF fusion protein showed greater cytotoxicity in various cancer cell lines compared to natural LDM and exerted a striking therapeutic effect against lung carcinoma xenografts using EGFR overexpressing A549 cells in athymic mice. At $0.24 \mathrm{mg} / \mathrm{kg}$, Ec-LDP(AE)-DF inhibited tumor growth by $77.4 \%$. Moreover, Ec-LDP(AE)-DF also showed potent therapeutic effects in xenografts using H460 cells, which express low levels of EGFR, with treatment inhibiting tumor growth by $76.2 \%$. Treatment with the AE-activated fusion protein also inhibited the activation of EGFR. No histopathological changes were found in various organs of the treated animals, suggesting that the effective dosage was well tolerated. Taken together, it can be concluded that the application of HBD1 as an immunomodulatory molecule for targeted drug therapy is not only feasible, but could be a powerful clinical treatment option. To elucidate the mechanisms of Ec-LDP(AE)-DF exhibited cytotoxicity on NSCLC cancer cells, PI staining study was also performed to examine cell cycle arrest. Results from cell cycle analysis demonstrated that Ec-LDP(AE)-DF caused significant G2-M arrest after treatment with 0.5 and $1 \mathrm{nmol} / \mathrm{L}$ Ec-LDP(AE)-DF. Ec-LDP(AE)-DF also induced apoptosis in NSCLC cells in a dosage-dependent manner, and the apoptosis induced by Ec-LDP(AE)-DF may be associated with mitochondrial pathways, because Bax activities as well as PARP cleavage increased significantly, and Bcl-2 activities decreased as shown by the Western blot analysis (Fig. S2).

In summary, this study used a novel EGFR-directed, LDM-activated fusion protein to target and modulate HBD1 expression as a cancer cell therapy using both NSCLC cells in vitro and in vivo tumor xenografts. To our knowledge, this analysis of the properties of the recombinant fusion protein, including its EGFR targeting capability in NSCLC cells, antitumor efficacy, and related mechanism of action, is the first of its kind. Although a wide variety of cancer therapies are currently in use or under investigation, the potential application of the novel HBD1 fusion protein described here is promising. As the role of defensins in cancer development and progression becomes clearer, defensin-based treatments will only become more essential and useful.

Acknowledgements This study was financially supported by the HighTech Research and Development Program of Shandong Province (2014GSF118022); the National Natural Science Foundation of China (Nos. 81502691 and 81573246); the China Postdoctoral Science Foundation Grant (2017M622188); and the Natural Science Foundation of Shandong Province (ZR2015HM040).

\section{Compliance with ethical standards}

Conflict of interest The authors declare that they have no conflict of interest.

\section{References}

1. Jarczak J, Kosciuczuk EM, Lisowski P, Strzalkowska N, Jozwik A, Horbanczuk J, et al. Defensins: natural component of human innate immunity. Hum Immunol. 2013;74:1069-79.

2. Ulm H, Wilmes M, Shai Y, Sahl HG. Antimicrobial host defensins-specific antibiotic activities and innate defense modulation. Front Immunol. 2012;3:249.

3. Yang D, Chertov O, Bykovskaia SN, Chen Q, Buffo MJ, Shogan $\mathrm{J}$, et al. Beta-defensins: linking innate and adaptive immunity through dendritic and T cell CCR6. Science. 1999;286:525-8.

4. Papo N, Shai Y. Host defense peptides as new weapons in cancer treatment. Cell Mol Life Sci. 2005;62:784-90.

5. Droin N, Hendra JB, Ducoroy P, Solary E. Human defensins as cancer biomarkers and antitumour molecules. J Proteomics. 2009;72:918-27.

6. Prado-Montes DOE. Human beta-defensin 1: a restless warrior against allergies, infections and cancer. Int J Biochem Cell Biol. 2010;42:800-4.

7. Sun CQ, Arnold R, Fernandez-Golarz C, Parrish AB, Almekinder $\mathrm{T}$, He J, et al. Human beta-defensin-1, a potential chromosome $8 \mathrm{p}$ tumor suppressor: control of transcription and induction of apoptosis in renal cell carcinoma. Cancer Res. 2006;66:8542-9.

8. Phan TK, Lay FT, Poon IK, Hinds MG, Kvansakul M, Hulett MD. Human beta-defensin 3 contains an oncolytic motif that binds PI $(4,5) \mathrm{P} 2$ to mediate tumour cell permeabilisation. Oncotarget. 2016;7:2054-69.

9. Shao RG, Zhen YS. Enediyne anticancer antibiotic lidamycin: chemistry, biology and pharmacology. Anticancer Agents Med Chem. 2008;8:123-31.

10. Ru Q, Shang BY, Miao QF, Li L, Wu SY, Gao RJ, et al. A cell penetrating peptide-integrated and enediyne-energized fusion protein shows potent antitumor activity. Eur J Pharm Sci. 2012;47:781-9.

11. Guo XF, Zhu XF, Shang Y, Zhang SH, Zhen YS. A bispecific enediyne-energized fusion protein containing ligand-based and antibody-based oligopeptides against epidermal growth factor receptor and human epidermal growth factor receptor 2 shows potent antitumor activity. Clin Cancer Res. 2010;16:2085-94.

12. Zhong G, Zhang S, Li Y, Liu X, Gao R, Miao Q, et al. A tandem $\mathrm{scFv}$-based fusion protein and its enediyne-energized analogue show intensified therapeutic efficacy against lung carcinoma xenograft in athymic mice. Cancer Lett. 2010;295:124-33.

13. Guo XF, Zhu XF, Cao HY, Zhong GS, Li L, Deng BG, et al. A bispecific enediyne-energized fusion protein targeting both epidermal growth factor receptor and insulin-like growth factor 1 receptor showing enhanced antitumor efficacy against non-small cell lung cancer. Oncotarget. 2017;8:27286-99.

14. Sun YW, Xu J, Zhou J, Liu WJ. Targeted drugs for systemic therapy of lung cancer with brain metastases. Oncotarget. 2018;9: 5459-72.

15. Liu WJ, Liu XJ, Li L, Li Y, Zhang SH, Zhen YS. Tuftsin-based, EGFR-targeting fusion protein and its enediyne-energized analog show high antitumor efficacy associated with CD47 downregulation. Cancer Immunol Immunother. 2014;63:1261-72.

16. Liu W-J, Liu X-J, Xu J, Li L, Li Y, Zhang S-H, et al. EGFRtargeting, $\beta$-defensin-tailored fusion protein exhibits high therapeutic efficacy against EGFR-expressed human carcinoma via mitochondria-mediated apoptosis. Acta Pharmacologica Sinica. 2018;1-10. https://doi.org/10.1038/s41401-018-0069-8. 
17. Xu J, Liu XJ, Li L, Zhang SH, Li Y, Gao RJ, et al. An engineered TIMP2-based and enediyne-integrated fusion protein for targeting MMP-14 shows potent antitumor efficacy. Oncotarget. 2015;6: 26322-34.

18. Xu J, Du Y, Liu XJ, Zhu BY, Zhang SH, Li L, et al. Recombinant EGFR/MMP-2 bi-targeted fusion protein markedly binding to non-small-cell lung carcinoma and exerting potent therapeutic efficacy. Pharmacol Res. 2017;126:66-76.

19. Wang X, Qiao X, Shang Y, Zhang S, Li Y, He H, et al. RGD and NGR modified TRAIL protein exhibited potent anti-metastasis effects on TRAIL-insensitive cancer cells in vitro and in vivo. Amino Acids. 2017;49:931-41.

20. Du Y, Shang BY, Sheng WJ, Zhang SH, Li Y, Miao QF, et al. A recombinantly tailored beta-defensin that displays intensive macropinocytosis-mediated uptake exerting potent efficacy against K-Ras mutant pancreatic cancer. Oncotarget. 2016;7: 58418-34.

21. Suarez-Carmona M, Hubert P, Delvenne P, Herfs M. Defensins: "Simple" antimicrobial peptides or broad-spectrum molecules? Cytokine Growth Factor Rev. 2015;26:361-70.

22. Zhuravel OV, Gerashchenko OL, Khetsuriani MR, Soldatkina MA, Pogrebnoy PV. Expression of human beta-defensins-1-4 in thyroid cancer cells and new insight on biologic activity of hBD-2 in vitro. Exp Oncol. 2014;36:174-8.

23. Zhang M, Qiu Z, Li Y, Yang Y, Zhang Q, Xiang Q, et al. Construction and characterization of a recombinant human beta defensin 2 fusion protein targeting the epidermal growth factor receptor: in vitro study. Appl Microbiol Biotechnol. 2013;97: 3913-23.

24. Wilmes M, Sahl HG. Defensin-based anti-infective strategies. Int J Med Microbiol. 2014;304:93-9.

25. Semlali A, Al Amiri A, Azzi A, Al Shahrani O, Arafah M, Kohailan M, et al. Expression and new exon mutations of the human beta defensins and their association on colon cancer development. PLoS One. 2015;10:e126868.
26. Kamino $\mathrm{Y}$, Kurashige $\mathrm{Y}$, Uehara O, Sato J, Nishimura $\mathrm{M}$, Yoshida $\mathrm{K}$, et al. HBD-2 is downregulated in oral carcinoma cells by DNA hypermethylation, and increased expression of hBD-2 by DNA demethylation and gene transfection inhibits cell proliferation and invasion. Oncol Rep. 2014;32:462-8.

27. Baxter AA, Poon IK, Hulett MD. The plant defensin NaD1 induces tumor cell death via a non-apoptotic, membranolytic process. Cell Death Discov. 2017;3:16102.

28. Xu J, Du Y, Liu W-J, Li L, Li Y, Wang X-F, et al. Intensive fibrosarcoma-binding capability of the reconstituted analog and its antitumor activity. Taylor \& Francis 2017. https://doi.org/10. 1080/10717544.2017.1410261.

29. Roskoski RJ. The ErbB/HER receptor protein-tyrosine kinases and cancer. Biochem Biophys Res Commun. 2004;319:1-11.

30. Kokai Y, Myers JN, Wada T, Brown VI, LeVea CM, Davis JG, et al. Synergistic interaction of p185c-neu and the EGF receptor leads to transformation of rodent fibroblasts. Cell. 1989;58: 287-92.

31. Wen R, Umeano AC, Dhar S. Accessing mitochondrial targets using NanoCargos. In: Intracellular delivery III. Springer; 2016. p. $229-54$.

32. Guo XF, Zhu XF, Yang WC, Zhang SH, Zhen YS. An EGFR/ HER2-bispecific and enediyne-energized fusion protein shows high efficacy against esophageal cancer. PLoS ONE. 2014;9: e92986.

33. Janthur WD, Cantoni N, Mamot C. Drug conjugates such as Antibody Drug Conjugates (ADCs), immunotoxins and immunoliposomes challenge daily clinical practice. Int $\mathrm{J}$ Mol Sci. 2012;13:16020-45.

34. Pathak RK, Wen R, Kolishetti N, Dhar S. A prodrug of two approved drugs, cisplatin and chlorambucil, for chemo war against cancer. Mol Cancer Ther. 2017;16:625-36.

35. Wen R, Banik B, Pathak RK, Kumar A, Kolishetti N, Dhar S. Nanotechnology inspired tools for mitochondrial dysfunction related diseases. Adv Drug Deliv Rev. 2016;99:52-69. 